\title{
Output Feedback Performance Recovery in the Presence of Uncertainties th
}

\author{
Joonho Lee ${ }^{\mathrm{a}, *}$, Ranjan Mukherjee ${ }^{\mathrm{a}}$, Hassan K. Khalil ${ }^{\mathrm{b}}$ \\ ${ }^{a}$ Department of Mechancial Engineering, Michigan State University, East Lansing, MI 48824, USA \\ ${ }^{b}$ Department of Electrical and Computer Engineering, Michigan State University, East Lansing, MI 48824, USA
}

\begin{abstract}
We propose an output-feedback tracking controller for uncertain, nonaffine, nonlinear systems. The output feedback controller results in a closed-loop system with a three-time-scale structure; an extended high-gain observer estimates unmeasured states and uncertainties in the fastest time scale and dynamic inversion is used to deal with nonaffine inputs and input uncertainties in the intermediate time scale while the plant dynamics evolves in the slowest time scale. The dynamic inversion algorithm is based on sector conditions and results in exponential convergence of the inputs. Together with the extended high-gain observer, dynamic inversion results in performance recovery of a target system. The singular perturbation method is used to analyze the stability and performance of the system and numerical simulations are used to demonstrate the effectiveness of the control design.
\end{abstract}

Keywords: Extended High-Gain Observers; Dynamic inversion; Uncertain nonlinear systems; Performance recovery

\section{Introduction}

Control of dynamical systems is challenging in the presence of uncertainties. Uncertainties may arise from inaccurate description of the dynamics by the mathematical model used, or can be due to external disturbances that are not accounted for in the model. Additional challenges are posed when the states of the system are not available for measurement and the control variables do not appear linearly in the mathematical model. To achieve desired control objectives, many of these challenges have been addressed by several researchers.

To consider uncertain, nonaffine systems with external disturbances, Chakrabortty and Arcak (2009a,b) designed a timescale separation method. Two filters were used in Chakrabortty and Arcak (2009b) to deal with system uncertainties and the nonaffine input; one filter was designed to estimate the uncertainties and the other filter was used to deal with the nonaffine input. The work in Chakrabortty and Arcak (2009a) is an extension of Chakrabortty and Arcak (2009b) where unmodeled dynamics in the control inputs are additionally taken into account. Hovakimyan et al. (2008b) proposed a fast gradient algorithm for dynamic inversion to deal with Multi-Input-Multi-Output (MIMO) nonaffine systems. The control approaches developed by Chakrabortty and Arcak (2009a,b) and Hovakimyan et al. (2008b) are based on state feedback.

Tanelli et al. (2012) designed a state feedback control scheme for uncertain, Single-Input-Single-Output (SISO) nonaffine

\footnotetext{
The material in this paper was partially presented at the 51st IEEE Conference on Decision and Control (CDC), December 10-13, 2012, Maui, Hawaii, USA.

${ }^{*}$ Corresponding author

Email addresses: lee joon8@msu .edu (Joonho Lee), mukherji@egr.msu. edu (Ranjan Mukherjee), khalilemsu.edu (Hassan K. Khalil)
}

systems. A second-order sliding mode controller with saturation was used to deal with uncertainties and the nonaffine input. The system states were estimated using the first-order differentiator proposed by Lavant (1998) but the stability analysis was based on state feedback control.

In Freidovich and Khalil (2008), Extended High-Gain Observers (EHGOs) were designed to estimate unmeasured states and uncertainties by combining the High-Gain Observers (HGOs) proposed by Atassi and Khalil (1999) with an additional integrator. Back and Shim (2008) developed controllers for uncertain, SISO nonline systems using a time-scale separation approach and the circle criterion; the unmeasured states and external disturbances were estimated using an observer. These results were extended in Back and Shim (2009) to deal with uncertain MIMO systems using the multi-variable circle criterion. The results in Freidovich and Khalil (2008) and Back and Shim $(2008,2009)$ are applicable to systems with affine inputs.

Hovakimyan et al. (2008a) proposed an output feedback controller for uncertain, SISO nonaffine input systems using neural network observers together with dynamic inversion. Ge and Zhang (2003) used neural networks to deal with SISO nonaffine systems and high-gain observers were used to estimate the unmeasured states. Chen et al. (2010) proposed state feedback control for uncertain, MIMO nonaffine systems using neural networks. Neural networks were used to model the system dynamics, uncertainties in the system, and input nonlinearities and uncertainties. A robust backstepping controller, combining backstepping with variable structure control, was used to deal with neural networks approximation errors. All of these methods based on neural networks require heavy computations and good prior knowledge of the system.

In this paper, we propose an output feedback control scheme for uncertain nonlinear systems with nonaffine inputs and exter- 
nal disturbances; it is an extension of our earlier work Lee et al. (2012a,b). By operating an EHGO in the fastest time scale, unmeasured system states, model uncertainties, and external disturbance were estimated. For the fast recovery of target system inputs in the presence of uncertainties, dynamic inversion was used based on the estimates provided by the EHGO together with sector conditions for the inputs. Using a multi-time-scale controller, the performance of target system states and inputs is recovered in the presence of uncertainties. The singular perturbation method is used to analyze the closed-loop system behavior and establish stability and performance recovery.

This paper is organized as follows. In Section 2, the problem is formulated for uncertain MIMO systems. In Section 3, the output feedback controller is presented along with the EHGO and the dynamic inversion algorithm. Section 4 provides the stability analysis and establishes performance recovery. Simulation results are presented in Section 5 and compared with results of other algorithms in the literature. Performance recovery is also verified through simulation. Concluding remarks are provided in Section 6.

\section{Problem Statements}

The goal of this paper is to design an output feedback controller that can make an uncertain MIMO nonlinear system follow a target system. Consider a MIMO nonlinear system given by

$$
\begin{aligned}
\dot{x} & =A x+B f(x, z, u), \quad x(0)=x_{0} \\
\dot{z} & =f_{0}(x, z), \quad z(0)=z_{0} \\
y & =C x,
\end{aligned}
$$

where $A, B$, and $C$ represent n chains of integrators as

$$
\begin{aligned}
& A=\text { block } \operatorname{diag}\left[A_{1}, \ldots, A_{n}\right], B=\text { block } \operatorname{diag}\left[B_{1}, \ldots, B_{n}\right] \\
& A_{i}=\left[\begin{array}{ccccc}
0 & 1 & 0 & \cdots & 0 \\
0 & 0 & 1 & \cdots & 0 \\
\vdots & \vdots & \ddots & \ddots & \vdots \\
0 & 0 & \cdots & 0 & 1 \\
0 & 0 & \cdots & \cdots & 0
\end{array}\right]_{\rho_{i} \times \rho_{i}}, B_{i}=\left[\begin{array}{c}
0 \\
0 \\
\vdots \\
0 \\
1
\end{array}\right]_{\rho_{i} \times 1} \\
& C=\text { block } \operatorname{diag}\left[C_{1}, \ldots, C_{n}\right], C_{i}=[1,0, \cdots, 0]_{1 \times \rho_{i}},
\end{aligned}
$$

and $f(x, z, u)=f_{n}(x, u)+\sigma(x, z, u)$ with

$$
f_{n}(x, u)=\left[\begin{array}{c}
f_{n, 1} \\
\vdots \\
f_{n, n}
\end{array}\right]_{n \times 1}, \sigma(x, z, u)=\left[\begin{array}{c}
\sigma_{1}(x, z, u) \\
\vdots \\
\sigma_{n}(x, z, u)
\end{array}\right]_{n \times 1}
$$

$1 \leq i \leq n, \rho=\rho_{1}+\cdots+\rho_{n}, x \in D_{x} \subset \mathbf{R}^{\rho}, z \in D_{z} \subset \mathbf{R}^{m}$, and $u \in D_{u} \subset \mathbf{R}^{n}$. The domains $D_{x}, D_{z}$, and $D_{u}$ contains their respective origins. The output $y \in D_{y} \subset \mathbf{R}^{n}$ is measured, the nominal function $f_{n}(x, u)$ is known, and $\sigma(x, z, u)$ is unknown.

In this paper, we are designing controllers for minimumphase systems. We make the following two assumptions.

Assumption 1. The functions $f_{n}(x, u), \sigma(x, z, u)$, and $f_{0}(x, z)$ are continuously differentiable.
Assumption 2. There exists a positive definite function $V_{z}$ such that for all $x \in D_{x}$ and $z \in D_{z}$

$$
\begin{aligned}
& \alpha_{1}(\|z\|) \leq V_{z}(z) \leq \alpha_{2}(\|z\|) \\
& \dot{V}_{z} \leq 0 \text { for }\|z\| \geq \alpha_{3}(\|x\|)
\end{aligned}
$$

where $\alpha_{1}(x), \alpha_{2}(x)$, and $\alpha_{3}(x)$ are class $\kappa$ functions.

Assumption 2 implies that the $z$-subsystem in (1) is boundedinput-bounded-state stable.

A target system is defined by

$$
\dot{x}_{r}=(A-B L) x_{r}+B u_{c}(t), \quad x_{r}(0)=x_{r 0},
$$

where the matrix $L$ is block diagonal such that the matrix $(A-$ $B L)$ is Hurwitz; $u_{c}(t)$ is a bounded command input belonging to the compact set $D_{r} \subset \mathbf{R}^{n}$; its derivative $\dot{u}_{c}$ is chosen to be bounded; and $x_{r}$ belongs to a compact set $D_{x_{r}}$ where $D_{x_{r}}$ is in the interior of $D_{x}$. With the variable $e=x-x_{r}$, the error dynamics are given by

$$
\dot{e}=(A-B L) e+B F\left(x, z, u, u_{c}\right), \quad e_{0}=e(0)
$$

where $F\left(x, z, u, u_{c}\right)=f(x, z, u)+L x-u_{c}(t)$. With the error dynamics of (3), we have the following assumption.

Assumption 3. There is a unique continuously differentiable function $\phi\left(x, z, u_{c}\right)$ such that $u_{r}=\phi\left(x, z, u_{c}\right)$ solves the equation

$$
F\left(x, z, u_{r}, u_{c}\right)=0
$$

The derivative $\dot{u}_{r}=\phi_{d}\left(x, z, u_{c}, \dot{u}_{c}\right)$ is bounded on compact sets of $x$ and $z$. Furthermore, there is a known matrix $K\left(x, s+u_{r}, u_{c}\right)$ such that the function $F$ satisfies the sector condition

$$
s^{T} K\left(x, s+u_{r}, u_{c}\right) F\left(x, z, s+u_{r}, u_{c}\right) \geq \beta s^{T} s, \quad \beta>0
$$

for all $\left(x, z, u_{d}\right)$, with $s=u-u_{r}$.

Remark 1. When the system is affine in $u$, i.e., $f(x, z, u)=$ $h(x, z)+G(x, z) u$, condition (5) is equivalent to the existence of a known matrix $K\left(x, s+u_{r}, u_{c}\right)$ such that

$$
K\left(x, s+u_{r}, u_{c}\right) G(x, z)+G^{T}(x, z) K^{T}\left(x, s+u_{r}, u_{c}\right) \geq k I
$$

with $k>0$. This condition holds with $K=I$ when $G(x, z)$ satisfies

$$
G(x, z)+G^{T}(x, z) \geq k I, \quad k>0
$$

This will be the case for single-input systems when $G$ is positive and bounded away from zero. Condition (6) is less restrictive than (7) as it will be shown in Section 5 by an example.

Remark 2. Earlier work on systems that are nonaffine in the input, in particular, Chakrabortty and Arcak (2009b) requires the Jacobian matrix $(\partial f / \partial u)$ to satisfy the condition.

$$
\left(\frac{\partial f}{\partial u}\right)+\left(\frac{\partial f}{\partial u}\right)^{T} \geq k I, \quad k>0
$$


and Hovakimyan et al. $(2005,2008 a, b)$ require the Jacobian matrix $(\partial f / \partial u)$ to satisfy either the condition (8) or the condition

$\zeta^{T}\left(\frac{\partial f^{T}}{\partial u}\left(x, u_{1}, u_{c}\right)\right)\left(\frac{\partial f}{\partial u}\left(x, u_{2}, u_{c}\right)\right) \zeta \geq 2 k_{c}\|\zeta\|^{2} \quad \forall \zeta \in \mathbf{R}^{n}$

where $u_{1}$ and $u_{2}$ are distinct variables and $k_{c}$ is a positive constant. The sector condition (5) is less restrictive than (8) and (9). For single-input systems, (8) requires the Jacobian to be positive for all $u$. The sector condition (5), on the other hand, allows the Jacobian to be negative as long as $f(x, z, u)$ belongs to the sector $[k, \infty)$, uniformly in $x, z$ and $u_{c}$.

For multi-input systems, the sector condition does not require the Jacobian $(\partial f / \partial u)$ to be nonsingular. In Section 5, it is shown by examples that the sector condition in (6) is less conservative than the conditions (8) and (9).

\section{Control Design}

We use dynamic inversion to deal with nonaffine and/ or uncertain functions. Define $F_{c}\left(x, \sigma, u, u_{c}\right)$ by

$$
\begin{aligned}
F\left(x, z, u, u_{c}\right) & =f(x, z, u)+L x-u_{c} \\
& =f_{n}(x, u)+\sigma+L x-u_{c} \\
& \triangleq F_{c}\left(x, \sigma, u, u_{c}\right)
\end{aligned}
$$

If $x$ and $\sigma$ are available, the dynamic inversion can be

$$
\mu \dot{u}=-K\left(x, u, u_{c}\right) F_{c}\left(x, \sigma, u, u_{c}\right), \quad u(0)=u_{0}
$$

where $\mu$ is a small positive constant.

Let us consider closed-loop system under the state feedback

$$
\begin{aligned}
\dot{e} & =(A-B L) e+B F\left(x, z, s+u_{r}, u_{c}\right), \\
\dot{z} & =f_{0}(x, z), \\
\mu \dot{s} & =-K F\left(x, z, s+u_{r}, u_{c}\right)-\mu \phi_{d}
\end{aligned}
$$

Let $V_{s}=s^{T} s / 2$ and $V_{e}=e^{T} P e$, where $P$ is the solution of the Lyapunov equation $P(A-B L)+(A-B L)^{T} P=-I$ and $I$ is the identity matrix. From (5) and Assumption 3,

$$
\begin{aligned}
\dot{V}_{s} & \leq-\left(\frac{\beta}{\mu}\right)\|s\|^{2}+2\|s\|\left\|\phi_{d}\right\| \\
\dot{V}_{e} & =-e^{T} e+2 e^{T} B F\left(x, z, s+u_{r}, u_{c}\right) \\
& \leq-\|e\|^{2}+k_{e}\|e\|\|s\|
\end{aligned}
$$

on the compact sets, for sufficiently small $\mu$. Therefore, there exist constants $b_{1}>0, b_{2}>0$, and $c_{0}>0$ such that

$$
\Omega_{b}=\left\{V_{e} \leq b_{1}\right\} \times\left\{V_{z} \leq c_{0}\right\} \times\left\{V_{s} \leq b_{2}\right\}
$$

is compact, positively invariant with respect to (11) and $\forall(e, z, s) \in \Omega_{b}, x \in D_{x}, z \in D_{z}$, and $u \in D_{u}$.
In output feedback control, $x$ and $\sigma$ are estimated using the EHGO:

$$
\begin{aligned}
& \dot{\hat{x}}=A \hat{x}+B\left[f_{n}(\hat{x}, u)+\hat{\sigma}(t)\right]+H(\varepsilon)(y-C \hat{x}) \\
& \dot{\hat{\sigma}}=H_{n+1}(y-C \hat{x}), \quad \hat{x}(0)=\hat{x}_{0}, \quad \hat{\sigma}(0)=\hat{\sigma}_{0}
\end{aligned}
$$

where $\hat{\sigma}(t)=\left[\hat{\sigma}_{1}, \cdots, \hat{\sigma}_{n}\right]^{T} \in \mathrm{R}^{n}$ is the estimate of $\sigma(x, z, u)$, and

$$
\begin{aligned}
& H=\text { block } \operatorname{diag}\left[H_{1}, \cdots, H_{n}\right], \\
& H_{i}(\varepsilon)=\left[\alpha_{i, 1} / \varepsilon, \cdots, \alpha_{i, \rho_{i}} / \varepsilon^{\rho_{i}}\right]^{T}, \\
& H_{n+1}=\left[\alpha_{1, \rho_{1}} / \varepsilon^{\rho_{1}+1}, \cdots, \alpha_{n, \rho_{n}} / \varepsilon^{\rho_{n}+1}\right]^{T}
\end{aligned}
$$

The constants $\alpha_{i, 1}, \cdots, \alpha_{i, \rho_{i}+1}$ are chosen such that the polynomials

$$
\lambda^{\rho_{i}+1}+\alpha_{i, 1} \lambda^{\rho_{i}}+\cdots+\alpha_{i, \rho_{i}} \lambda+\alpha_{i, \rho_{i}+1} \quad \text { for } i=1, \ldots, n
$$

are Hurwitz and the parameter $\varepsilon>0$ is small enough. We note that $\varepsilon$ is smaller than $\mu$ to make the EHGO faster than the dynamic inversion since the dynamic inversion uses estimates provided by the EHGO. Therefore, the parameters $\varepsilon$ and $\mu$ are chosen such that $0<\varepsilon \ll \mu \ll 1$.

Using the EHGO in (14) together with the dynamic inversion in (10), the output feedback control is designed as

$$
\begin{aligned}
& \mu \dot{u}=-K\left(\hat{x}_{s}, u, u_{c}\right)\left[f_{n}\left(\hat{x}_{s}, u\right)+\hat{\sigma}_{s}+L \hat{x}_{s}-u_{c}\right] \\
& \hat{x}_{s}=\left[\hat{x}_{s 1}^{T}, \ldots, \hat{x}_{s n}^{T}\right]^{T}, \quad \hat{\sigma}_{s}=\left[\hat{\sigma}_{s 1}, \ldots, \hat{\sigma}_{s n}\right]^{T} \\
& \hat{x}_{s i}=\left[M_{x_{i, 1}} \operatorname{sat}\left(\frac{\hat{x}_{i, 1}}{M_{x_{i, 1}}}\right), \ldots, M_{x_{i, \rho_{i}}} \operatorname{sat}\left(\frac{\hat{x}_{i, \rho_{i}}}{M_{x_{i, \rho_{i}}}}\right)\right]^{T} \\
& \hat{\sigma}_{s i}=\left[M_{\sigma_{1}} \operatorname{sat}\left(\frac{\hat{\sigma}_{1}}{M_{\sigma_{1}}}\right), \ldots, M_{\sigma_{n}} \operatorname{sat}\left(\frac{\hat{\sigma}_{n}}{M_{\sigma_{n}}}\right)\right]^{T}
\end{aligned}
$$

for $i=1, \ldots, n$, where $\operatorname{sat}(\cdot)$ is the saturation function defined by

$$
\operatorname{sat}(k)=\left\{\begin{array}{cc}
k & |k| \leq 1 \\
\operatorname{sign}(k) & |k|>1
\end{array}\right.
$$

The saturation function is used to prevent peaking from degrading the system performance. The saturation levels $M_{x_{i, j}}$ and $M_{\sigma_{i}}$ for $j=1, \ldots, \rho_{i}$ and $i=1, \ldots, n$ in (15), are chosen as

$$
M_{x_{i, j}}>\max \left|x_{i, j}\right|, \quad M_{\sigma_{i}}>\max \left|\sigma_{i}\left(e, z, s+u_{r}\right)\right|
$$

for $j=1, \ldots, \rho_{i}$ and $i=1, \ldots, n$, where maximization is taken over $\Omega_{b}$ in (13).

Remark 3. The procedure for choosing $\mu$ and $\varepsilon$ for a multitime-scale structure is provided as follows.

- First, under the state feedback, the control parameter, $\mu$ in the dynamic inversion is chosen to meet design criteria.

- Secondly, the saturation levels are chosen such that the saturation is not activated under the state feedback. 
- Thirdly, the Extended High-Gain Observer, which estimates unmeasured states and uncertainties, is designed by choosing $0<\varepsilon \ll \mu \ll 1$.

- Lastly, the estimates from the Extended High-Gain Observer are saturated and then fed into the controller for the output feedback.

\section{Main Result}

In this section, we will show that in the presence of uncertainties, the output feedback control (14) and (15) can recover the performance of both states and inputs of the target system (2). Consider the fast variables $\eta=\left[\eta_{1}^{T}, \cdots, \eta_{n+1}^{T}\right]^{T}$ with $\eta_{i}=\left[\eta_{i, 1}, \cdots, \eta_{i, \rho_{i}}\right]^{T} \in \mathrm{R}^{\rho_{i}}$, and

$$
\begin{aligned}
& \eta_{i, j}=\frac{x_{i, j}-\hat{x}_{i, j}}{\varepsilon^{\rho_{i}+1-j}}, \quad \eta_{i, \rho_{i}+1}=\sigma_{i}(x, z, u)-\hat{\sigma}_{i}(t) \\
& \text { for } 1 \leq i \leq n, \quad 1 \leq j \leq \rho_{i}
\end{aligned}
$$

for error dynamics of the EHGO. The closed-loop system equations can be written in the standard singularly perturbed form

$$
\begin{aligned}
\dot{e} & =(A-B L) e+B\left[f\left(x, z, s+u_{r}\right)+L x-u_{c}(t)\right], \\
\dot{z} & =f_{0}(x, z) \\
\mu \dot{s} & =-K\left[f_{n}\left(\hat{x}_{s}, s+u_{r}\right)+\hat{\sigma}_{s}+L \hat{x}_{s}-u_{c}\right]-\mu \phi_{d} \\
\varepsilon \dot{\eta} & =\Lambda \eta+\varepsilon\left[\bar{B}_{1} \psi_{1}+\left(\bar{B}_{2} / \mu\right) \psi_{2}\right]
\end{aligned}
$$

where

$$
\begin{aligned}
\Lambda & =\operatorname{block} \operatorname{diag}\left[\Lambda_{1}, \cdots, \Lambda_{n}\right] \\
\Lambda_{i} & =\left[\begin{array}{ccccc}
-\alpha_{i, 1} & 1 & 0 & \cdots & 0 \\
-\alpha_{i, 2} & 0 & 1 & \ddots & 0 \\
\vdots & \vdots & \ddots & \vdots & \vdots \\
-\alpha_{i, \rho_{i}} & 0 & \cdots & 0 & 1 \\
-\alpha_{i, \rho_{i}+1} & 0 & \cdots & \cdots & 0
\end{array}\right]_{\rho_{i} \times \rho_{i}} \\
\bar{B}_{1} & =\operatorname{block} \operatorname{diag}\left[B_{1,1}, \cdots, B_{1, n}\right], \\
\bar{B}_{2} & =\operatorname{block} \operatorname{diag}\left[B_{2,1}, \cdots, B_{2, n}\right], \\
B_{1, i} & =\left[\begin{array}{c}
0 \\
B_{i}
\end{array}\right]_{\left(\rho_{i}+1\right) \times 1}, \quad B_{2, i}=\left[\begin{array}{c}
B_{i} \\
0
\end{array}\right]_{\left(\rho_{i}+1\right) \times 1}
\end{aligned}
$$

for $i=1, \ldots, n$ and the functions $\psi_{1}$ and $\psi_{2}$ are given by

$$
\begin{aligned}
\psi_{1}= & (1 / \varepsilon)\left[f_{n}\left(x, s+u_{r}\right)-f_{n}\left(\hat{x}_{s}, s+u_{r}\right)\right] \\
\psi_{2}= & \mu\left\{\left(\frac{\partial \sigma}{\partial x}\right)^{T}\left[A x+B f\left(x, z, s+u_{r}\right)\right]\right. \\
& \left.+\left(\frac{\partial \sigma}{\partial z}\right)^{T} f_{0}(x, z)\right\}-\left(\frac{\partial \sigma}{\partial u}\right)^{T} K F_{c s} \\
F_{c s}= & f_{n}\left(\hat{x}_{s}, s+u_{r}\right)+\hat{\sigma}_{s}+L \hat{x}_{s}-u_{c}
\end{aligned}
$$

We note that $u_{r}$ and $\phi_{d}\left(x, z, s+u_{r}\right)$ in (17) and (18), respectively, are mentioned in Assumption 3, the nominal function $f_{n}\left(x, s+u_{r}\right)$ is Lipschitz in its arguments so that the inequality $\left\|f_{n}\left(x, s+u_{r}\right)-f_{n}\left(\hat{x}_{s}, s+u_{r}\right)\right\| \leq \varepsilon k_{\psi_{1}}\|\eta\|$ is satisfied with $k_{\psi_{1}}$ independent of $\varepsilon$.

We are going to show the stability of the boundary layer and reduced systems in the closed-loop system (17)-(20). Since the $z$-subsystem of (18) with the input $x$ is bounded-input-boundedstate stable, we are focusing on the subsystems (17), (19), and (20).

Now, using the time-scale structure of the closed-loop system (17), (19), and (20), the stability analysis of each subsystem will be shown. By considering the subsystem (20) as the fast system and the other subsystems (17) and (19) as the slow system, the boundary layer system in (20) can be obtained by

$$
\varepsilon \dot{\eta}=\Lambda \eta, \quad \eta(0)=\eta_{0}
$$

Since the matrix $\Lambda$ is Hurwitz, the boundary layer system (21) is exponentially stable at the origin.

After the fast variable $\eta$ reaches its quasi-steady state, $\eta=0$, the reduced system for (17) and (19) is obtained by setting $\eta=$ 0 . In the reduced system, the dynamic inversion (19) is fast and the subsystem (17) is slow. The boundary layer system for (19) is given by

$$
\mu \dot{s}=-K F\left(x, z, s+u_{r}, u_{c}\right), \quad s(0)=s_{0}
$$

The stability of the boundary layer system (22) is investigated using the Lyapunov function $V_{s}=\left(s^{T} s\right) / 2$. With the sector condition (5), its derivative $\dot{V}_{s}$ along the trajectory (22) is

$$
\mu \dot{V}_{s}=-s^{T} K F \leq-\beta\|s\|^{2}
$$

Therefore, the boundary layer system (22) is exponentially stable at $s=0$.

The reduced system for (17) is obtained by setting $\varepsilon=0$, $\eta=0, \mu=0$, and $s=0$. With the input $u=u_{r}$ and $x=\hat{x}$, the reduced system for (17) is obtained as

$$
\dot{e}=(A-B L) e, \quad e(0)=e_{0}
$$

which is exponentially stable at $e=0$.

Let

$$
\Omega_{a}=\left\{V_{e} \leq a_{1}\right\} \times\left\{V_{z} \leq c_{0}\right\} \times\left\{V_{s} \leq a_{2}\right\}
$$

where

$$
0<a_{1}<b_{1}, \quad 0<a_{2}<b_{2}
$$

and $Q_{x}$ and $Q_{s}$ be any compact subsets of $\mathbf{R}^{\rho}$ and $\mathbf{R}^{n}$, respectively. The following theorem shows that all trajectories starting from $\Omega_{a} \times Q_{x} \times Q_{x}$ will be bounded and $(x, u)$ approach the trajectories of the target system (2) as $\mu$ and $(\varepsilon / \mu)$ tend to zero.

Theorem 4.1. Consider the closed-loop system (17) - (20) under Assumption 1, 2, and 3. Suppose the trajectories $(e, z, s, \hat{x}, \hat{\sigma})$ start from the compact sets $\left(e_{0}, z_{0}, s_{0}\right) \in \Omega_{a}$ and $\left(\hat{x}_{0}, \hat{\sigma}_{0}\right) \in Q_{x} \times Q_{s}$. Then, there exists a constant $\varsigma>0$ such that for $\max \{\mu,(\varepsilon / \mu), \varepsilon\}<\varsigma$

- all trajectories are bounded; 


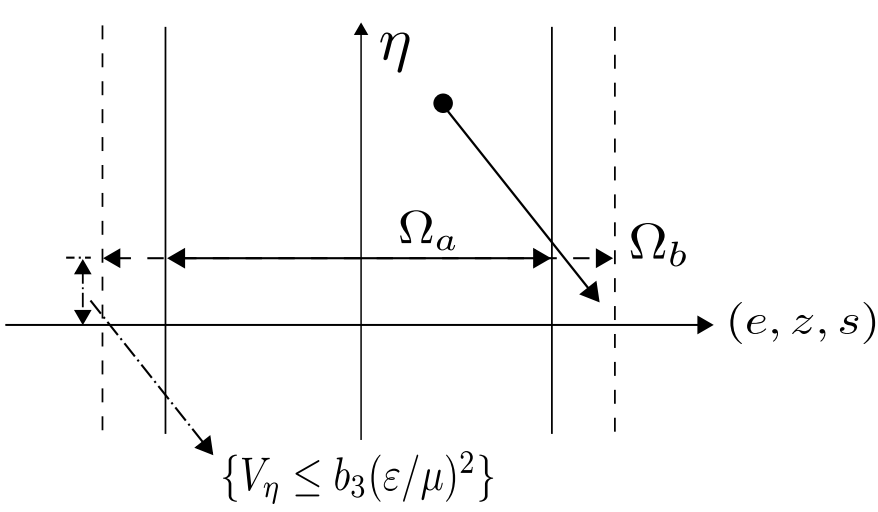

Figure 1: The trajectory starting from $\left(e_{0}, z_{0}, s_{0}\right) \in \Omega_{a}$ and $\eta_{0} \notin\left\{V_{\eta} \leq\right.$ $\left.b_{3}(\varepsilon / \mu)^{2}\right\}$ converges into $(e, z, s, \eta) \in \Omega_{b} \times\left\{V_{\eta} \leq b_{3}(\varepsilon / \mu)^{2}\right\}$.

- $\left\|x-x_{r}\right\| \rightarrow 0$ and $\left\|u-u_{r}\right\| \rightarrow 0$ as $\mu,(\varepsilon / \mu) \rightarrow 0$ for all $t \geq 0$.

Proof. As the first part of the proof, we are going to show that all trajectories enter a positively invariant set. We define the Lyapunov function $V_{\eta}=\eta^{T} P_{\eta} \eta$ for the subsystem, (20) where $P_{\eta}$ is the solutions for the Lyapunov equation $P_{\eta} \Lambda+\Lambda^{T} P_{\eta}=$ $-I$. Similar to earlier work on high-gain observers, e.g., Khalil (2002), it can be shown that, for sufficiently small $(\varepsilon / \mu)$, the set $\Omega_{b} \times\left\{V_{\eta} \leq b_{3}(\varepsilon / \mu)^{2}\right\}$, for some $b_{3}>0$, is positively invariant. This is done by showing that the derivatives of $V_{e}, V_{z}$, and $V_{s}$ are negative on the boundaries $\left\{V_{e}=b_{1}\right\},\left\{V_{z}=c_{0}\right\}$, and $\left\{V_{s}=b_{2}\right\}$, respectively. Similarly, it can be shown that for sufficiently small $\lambda=\max \{\mu, \varepsilon / \mu\}$, the set $\left\{V_{e} \leq b_{1}\right\} \times\left\{V_{z} \leq\right.$ $\left.c_{0}\right\} \times\left\{V_{s} \leq c_{2} \lambda^{2}\right\} \times\left\{V_{\eta} \leq b_{3}(\varepsilon / \mu)^{2}\right\}$, for some $c_{2}>0$, is positively invariant.

We show boundedness of the trajectories in two steps:

- firstly, the trajectories $(e, z, s)$ and $\eta$ starting from $\left(e_{0}, z_{0}, s_{0}\right) \in \Omega_{a}$ and $\eta(0) \notin\left\{V_{\eta} \leq b_{3}(\varepsilon / \mu)^{2}\right\}$ enter the set $\Omega_{b} \times\left\{V_{\eta} \leq b_{3}(\varepsilon / \mu)^{2}\right\}$ in finite time as depicted in Fig. 1;

- secondly, the trajectories $(e, z, s)$ and $\eta$ starting from $\Omega_{b} \times$ $\left\{V_{\eta} \leq b_{3}(\varepsilon / \mu)^{2}\right\}$ enters the set, $\left\{V_{e} \leq b_{1}\right\} \times\left\{V_{z} \leq c_{0}\right\} \times$ $\left\{V_{s} \leq c_{2} \lambda^{2}\right\} \times\left\{V_{\eta} \leq b_{3}(\varepsilon / \mu)^{2}\right\}$ in finite time.

In the first step, consider that the initial conditions are in $\left(e_{0}, z_{0}, s_{0}\right) \in \Omega_{a}$ and $\eta(0)$ starting from outside the set $\left\{V_{\eta} \leq\right.$ $\left.b_{3}(\varepsilon / \mu)^{2}\right\}$. Due to the scaling (16), $\eta(0)$ could be of the order of $1 / \varepsilon^{\rho_{m}}$ where $\rho_{m}=\max _{i=1, \ldots, n} \rho_{i}$. It can be shown that the term $\left[\bar{B}_{1} \psi_{1}+\left(\bar{B}_{2} / \mu\right) \psi_{2}\right]$ in $(20)$ is bounded by $k_{1}\|\eta\|+k_{2}$ for all $(e, z, s) \in \Omega_{b}$, for some positive constants $k_{1}$ and $k_{2}$, independent of $\varepsilon$. Therefore, it can be shown that

$$
\varepsilon \dot{V}_{\eta} \leq-\|\eta\|^{2}+\varepsilon k_{3}\|\eta\|^{2}+\varepsilon k_{4}\|\eta\|
$$

for some positive constants $k_{3}$ and $k_{4}$. Hence,

$$
\varepsilon \dot{V}_{\eta} \leq-\frac{1}{2}\|\eta\|^{2}+\frac{\varepsilon}{\mu}\|\eta\|
$$

for $\varepsilon<1 /\left(2 k_{3}\right)$. It follows that there is $b_{3}>0$ such that

$$
\varepsilon \dot{V}_{\eta} \leq-\gamma_{1} V_{\eta}, \quad \text { for } V_{\eta} \geq\left(\frac{\varepsilon}{\mu}\right)^{2} b_{3}
$$

for some $\gamma_{1}>0$. Taking into consideration that $\|\eta(0)\| \leq$ $k_{5} / \varepsilon^{\rho_{m}}$ for some positive constant $k_{5}>0$, we obtain

$$
V_{\eta} \leq \frac{k_{6}}{\varepsilon^{2 \rho_{m}}} e^{-\gamma_{1} t / \varepsilon}
$$

for some positive constant $k_{6}>0$. Since $\varepsilon<\varepsilon / \mu$ for $0<\varepsilon \ll$ $\mu \ll 1$, we can estimate the time the trajectory will enter $\left\{V_{\eta} \leq\right.$ $\left.b_{3}(\varepsilon / \mu)^{2}\right\}$ by the more conservative time, $T_{1}(\varepsilon)$, when $V_{\eta}=$ $b_{3} \varepsilon^{2}$. The time $T_{1}(\varepsilon)$ is given by

$$
\frac{k_{6}}{\varepsilon^{2 \rho_{m}}} e^{-\gamma_{1} T_{1} / \varepsilon}=b_{3} \varepsilon^{2} \Leftrightarrow T_{1}=\frac{\varepsilon}{\gamma_{1}} \ln \left(\frac{k_{6}}{b_{3} \varepsilon^{2\left(\rho_{m}+1\right)}}\right)
$$

By L'Hopital's rule, it can be shown that $\lim _{\varepsilon \rightarrow 0} T_{1}(\varepsilon)=0$. Because $a_{1}<b_{1}, a_{2}<b_{2}$, and the right-hand side functions of (17), (18), and (20) are bounded uniformly in $\varepsilon$, there is time $T_{0}$ such that $(e, z, s) \in \Omega_{b}$ for all $t \in\left[0, T_{0}\right]$. By choosing $\varepsilon$ small enough we can have $T_{1}(\varepsilon)=(1 / 2) T_{0}$.

In the second step, we repeat the same argument to show that $s$ enters the set $\left\{V_{s} \leq c_{2} \lambda^{2}\right\}$. The right-hand side of (19) is a perturbation of the right-hand side of (22) with the perturbation term bounded by $k_{7}\|\eta\|+k_{8} \mu$ for some positive constants $k_{7}$ and $k_{8}$. Because $\eta$ cannot leave the set $\left\{V_{\eta} \leq b(\varepsilon / \mu)^{2}\right\}$,

$$
k_{7}\|\eta\|+k_{8} \mu \leq k_{9} \lambda \quad \text { for some } k_{9}>0
$$

where $\lambda=\max \{\mu, \varepsilon / \mu\}$. Hence

$$
\mu \dot{V}_{s} \leq-\beta\|s\|^{2}+k_{10} \lambda\|s\|
$$

Therefore, there is $c_{2}>0$ such that

$$
\mu \dot{V}_{s} \leq-\gamma_{2} V_{s}, \quad \text { for } V_{s} \geq c_{2} \lambda^{2}
$$

This shows that there is time $T_{2}=T_{2}(\mu)$ such that the trajectory enters $\left\{V_{s} \leq c_{2} \lambda^{2}\right\}$. Once again by choosing $\mu$ small enough, $(e, z)$ stay in the set $\left\{V_{e} \leq b_{1}\right\} \times\left\{V_{z} \leq c_{0}\right\}$. Thus, with the time $T(\varepsilon, \mu)=T_{1}(\varepsilon)+T_{2}(\mu)$, the trajectory enters the set, $\left\{V_{e} \leq\right.$ $\left.b_{1}\right\} \times\left\{V_{z} \leq c_{0}\right\} \times\left\{V_{s} \leq c_{2} \lambda^{2}\right\} \times\left\{V_{\eta} \leq b_{3}(\varepsilon / \mu)^{2}\right\}$, which is positively invariant for sufficiently small $\mu$ and $\varepsilon / \mu$, in view of (12) and Assumption 2.

Lastly, we are going to show the performance recovery for $x$ and $u$. Since the proof is similar for both variables, we show it only for $u$. The nominal model of (19) is the system (22), which is exponentially stable at $s=0$. The difference between (19) and (22) is

$$
\begin{aligned}
G= & -K\left[f_{n}\left(\hat{x}_{s}, s+u_{r}\right)-f_{n}\left(x, s+u_{r}\right)\right. \\
& \left.+\hat{\sigma}_{s}(t)-\sigma\left(x, z, s+u_{r}\right)+L\left(\hat{x}_{s}-x\right)\right]+\mu \dot{u}_{r}
\end{aligned}
$$

where $\dot{u}_{r}=\phi_{d}\left(x, z, u_{c}, \dot{u}_{c}\right)$ is bounded on the compact set of $x$, $z, u_{c}$, and $\dot{u}_{c}$, i.e., $\left\|\dot{u}_{r}\right\| \leq u_{r m}$, with $u_{r m}>0$, uniformly in $\mu$ and $\varepsilon$. The function $G$ is bounded by

$$
\|G\| \leq K_{m}\left[\varepsilon L_{1}\|\eta\|+L_{2}\|\eta\|\right]+\mu u_{r m}
$$

for some positive constants $K_{m}, L_{1}, L_{2}$, which can be made arbitrarily small by choosing sufficient small $\mu$ and $\varepsilon / \mu$, for 
$t \geq T(\varepsilon, \mu)$. Using Theorem 9.1 in Khalil (2002), we conclude that

$$
\left\|u(t)-u_{r}(t)\right\| \leq \delta(\mu, \varepsilon / \mu), \quad \forall t \geq T(\mu, \varepsilon)>0
$$

where $\delta(\mu, \varepsilon) \rightarrow 0$ as $\mu,(\varepsilon / \mu) \rightarrow 0$. For the time interval $t \in$ $[0, T(\mu, \varepsilon)]$, since the trajectories $u$ and $u_{r}$ are in the compact set, we have two inequalities

$$
\|u(t)-u(0)\| \leq k_{t} t, \quad\left\|u_{r}(t)-u(0)\right\| \leq k_{t} t
$$

with $k_{t}>0$, during the time interval. Using the triangle inequality, we obtain the inequality

$$
\left\|u(t)-u_{r}(t)\right\| \leq 2 k_{t} T(\mu, \varepsilon), \quad \forall t \in[0, T(\mu, \varepsilon)]
$$

Therefore, given any $\delta_{1}>0$, we can ensure that

$$
\left\|u(t)-u_{r}(t)\right\| \leq \delta_{1}, \quad \text { for all } t \geq 0
$$

by choosing $\mu$ and $(\varepsilon / \mu)$ sufficiently small.

Remark 4. If all the assumption hold globally and $V_{z}$ is radially unbounded, i.e., $\alpha_{1}$ is a class $\kappa_{\infty}$ function, then the constants $a_{1}, a_{2}$, and $c_{0}$ can be chosen arbitrarily large. Hence the control can achieve semiglobal stabilization by choosing $\mu$, $\varepsilon / \mu$, and $\varepsilon$ sufficiently small.

\section{Simulations}

In this section, we choose examples for comparing our results with results presented in Hovakimyan et al. (2008b) and Chakrabortty and Arcak (2009b). The first example, which has nonaffine input, considers the case where the Jacobian matrix is singular at some values. As a second example, which has affine inputs and system uncertainties, a modified example in Chakrabortty and Arcak (2009b) is chosen to show that our proposed control methods provide less conservative conditions than ones in Chakrabortty and Arcak (2009b).

\subsection{Example 1}

First, we consider the case where the Jacobian matrix, $(\partial f / \partial u)$ in (9), is singular at some values. In this example, we assume that the state $x=\left[x_{1}, x_{2}\right]^{T}$ is available and there is no system uncertainty. The MIMO nonlinear nonaffine system is given by

$$
\left[\begin{array}{c}
\dot{x}_{1} \\
\dot{x}_{2}
\end{array}\right]=\left[\begin{array}{c}
x_{1}+2 u_{1}^{3}-15 u_{1}^{2}+36 u_{1} \\
2 u_{2}^{3}-15 u_{2}^{2}+36 u_{2}
\end{array}\right]
$$

The target system is

$$
\begin{aligned}
\dot{x}_{r} & =(A-B L) x_{r}+u_{c}, \quad x_{r}=\left[x_{r 1}, x_{r 2}\right]^{T} \\
u_{c} & =\left[\begin{array}{l}
100 \\
100
\end{array}\right], A-L B=\left[\begin{array}{cc}
-1 & 0 \\
0 & -1
\end{array}\right]
\end{aligned}
$$

Our proposed controller for the dynamic inversion is

$$
\mu \dot{u}=-\left[\begin{array}{c}
x_{1}+2 u_{1}^{3}-15 u_{1}^{2}+36 u_{1}+x_{1}-100 \\
2 u_{2}^{3}-15 u_{2}^{2}+36 u_{2}+x_{2}-100
\end{array}\right],
$$
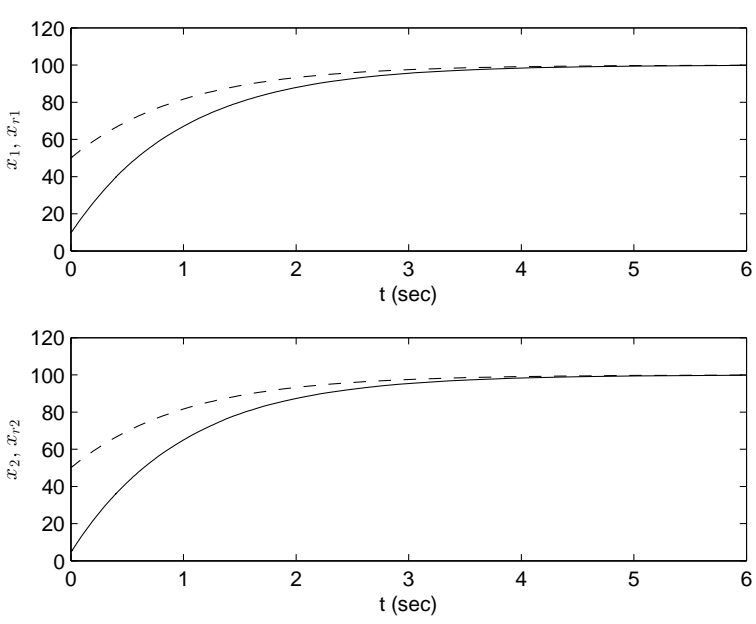

Figure 2: The solid and dashed lines represent trajectories $x_{i}$ for $i=1,2$ driven by the proposed controller in (24) and reference trajectories $x_{r i}$ for $i=1,2$ in (23), respectively.

where $u=\left[u_{1}, u_{2}\right]^{T}$, the small constant $\mu=0.1$, and $K$ in (5) is chosen as an identity matrix, i.e., $K=I_{2 \times 2}$ (a $2 \times 2$-identity matrix). In Fig. 2, the solid lines $x_{1}$ and $x_{2}$ generated by the proposed controller in (24), converge into the reference trajectories (dashed lines) in (23). Since the proposed controller in (24) is designed based on the sector condition, the controller is not affected by the singularity of the Jacobian matrix in Fig. 3.
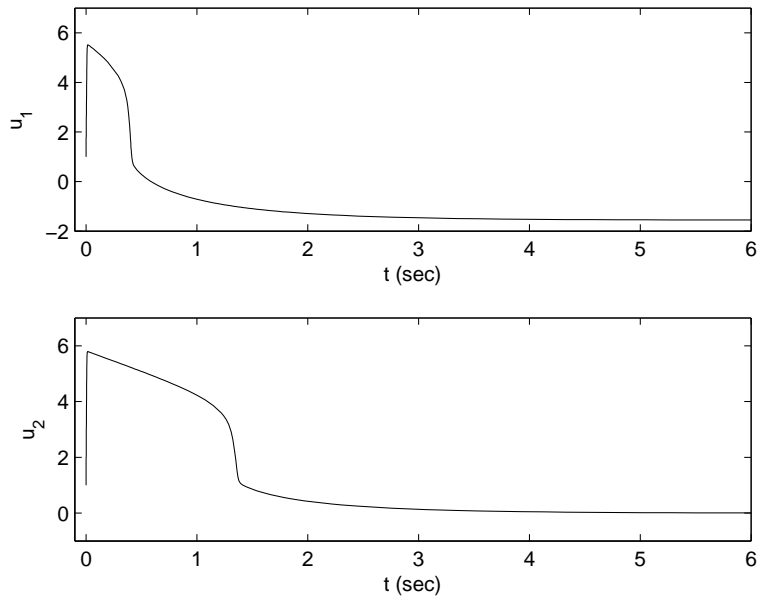

Figure 3: Input trajectories $u_{1}$ and $u_{2}$ in (24) are shown and both $u_{1}$ and $u_{2}$ cross the values $u_{1}=2,3$ and $u_{2}=2,3$ which make the Jacobian matrix $(\partial f / \partial u)$ singular.

\subsection{Example 2}

A modified model from Chakrabortty and Arcak (2009b) is given by

$$
\begin{aligned}
& \dot{x}=R_{m} u, \quad y=\left[x_{1}, x_{2}\right]^{T}, \\
& R_{m}=\left[\begin{array}{cc}
\cos \phi & \sin \phi \\
\sin \phi & -\cos \phi
\end{array}\right]
\end{aligned}
$$


with $x=\left[x_{1}, x_{2}\right]^{T}, u=\left[u_{1}, u_{2}\right]^{T}$, and $\phi=45^{\circ}$. The target system with $u_{c}=[0,0]^{T}$ is the same as in (23). With the condition $\phi=$ $45^{\circ}$, the Jacobian condition $\left(\partial R_{m} / \partial u\right)^{T}+\left(\partial R_{m} / \partial u\right) \geq k_{p} I_{2 \times 2}$ in Chakrabortty and Arcak (2009b) is not satisfied. Using the dynamic inversion, the control inputs are designed by

$$
\mu \dot{u}=-K F
$$

where

$$
K F=\left[\begin{array}{c}
u_{1} \cos \phi+u_{2} \sin \phi+x_{1} \\
-u_{1} \sin \phi+u_{2} \cos \phi-x_{2}
\end{array}\right]
$$

The sector condition in Assumption 3 is satisfied as follows.

$$
s^{T} K F \geq \beta\|s\|^{2}
$$

with the constant $\beta>0$. Now, the EHGO is designed to estimate uncertainties i.e., the right-hand side of (25), $R_{m} u$,

$$
\begin{array}{ll}
\dot{\hat{x}}_{1}=\hat{\sigma}_{1}+\frac{h_{11}}{\varepsilon}\left(x_{1}-\hat{x}_{1}\right), & \dot{\sigma}_{1}=\frac{h_{12}}{\varepsilon^{2}}\left(x_{1}-\hat{x}_{1}\right) \\
\dot{\hat{x}}_{2}=\hat{\sigma}_{2}+\frac{h_{21}}{\varepsilon}\left(x_{2}-\hat{x}_{2}\right), & \dot{\sigma}_{2}=\frac{h_{22}}{\varepsilon^{2}}\left(x_{2}-\hat{x}_{2}\right)
\end{array}
$$

where $\hat{\sigma}_{1}$ and $\hat{\sigma}_{2}$ are the estimates of $\dot{x}_{1}$ and $\dot{x}_{2}$, respectively. The constants $h_{i, 1}$ and $h_{i, 2}, i=1,2$ are chosen such that the polynomials

$$
\lambda^{2}+h_{i, 1} \lambda+h_{i, 2}=0, \quad \text { for } i=1,2
$$

are Hurwitz. The output feedback control is chosen as

$$
\mu \dot{u}=-K F_{c s}, \quad K F_{c s}=\left[\begin{array}{c}
M_{\sigma_{1}} \operatorname{sat}\left(\hat{\sigma}_{1} / M_{\sigma_{1}}\right)+x_{1} \\
-M_{\sigma_{2}} \operatorname{sat}\left(\hat{\sigma}_{2} / M_{\sigma_{2}}\right)-x_{2}
\end{array}\right]
$$

where the levels of saturation, $M_{\sigma_{1}}$ and $M_{\sigma_{2}}$ are chosen such that the saturation will not be activated in the range of state feedback control.

For the simulation, the parameters are given by

$$
\mu=0.02, \varepsilon=0.0002, h_{i, 1}=3, h_{i, 2}=1
$$

for $i=1,2$. In Fig. 4 and 5, the states $x_{1}$ and $x_{2}$ (solid lines) and their estimates $\hat{x}_{1}$ and $\hat{x}_{2}$ (dotted lines) are plotted. The initial conditions of trajectories are given by $x(0)=[2,4]^{T}, u(0)=$ $[0,0]^{T}$, the estimates $\hat{x}_{1}(0)=0$ and $\hat{x}_{2}(0)=0$, and $\hat{\sigma}_{1}(0)=0$ and $\hat{\sigma}_{2}(0)=0$. In Fig. 4 and 5, the estimate trajectories quickly converge into the system states and both the states and estimates are indistinguishable.
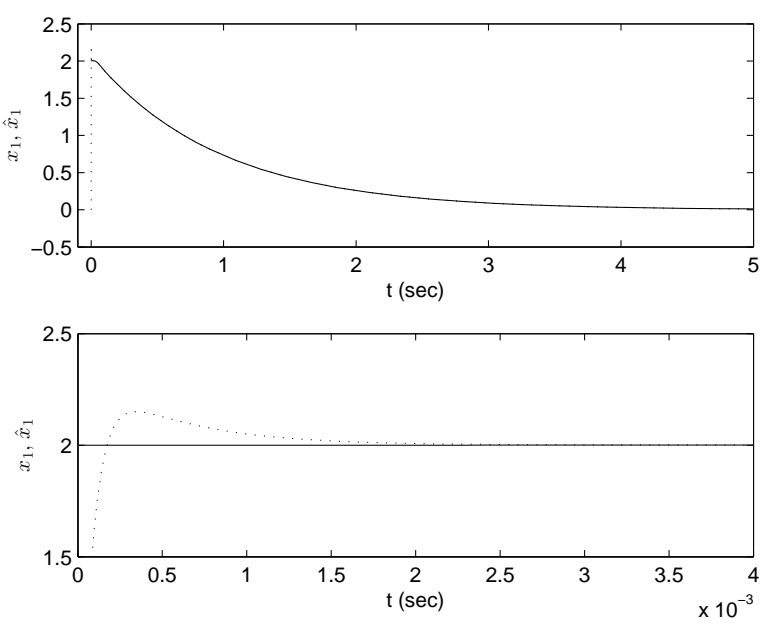

Figure 4: The solid and dotted lines represent the system state $x_{1}$ and estimate $\hat{x}_{1}$, respectively.
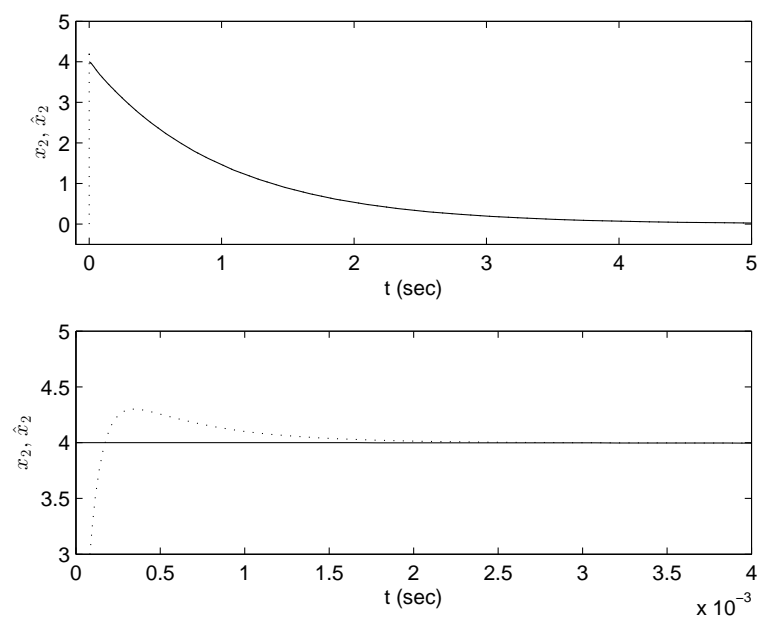

Figure 5: The solid and dotted lines represent the system state $x_{2}$ and estimate $\hat{x}_{2}$, respectively.
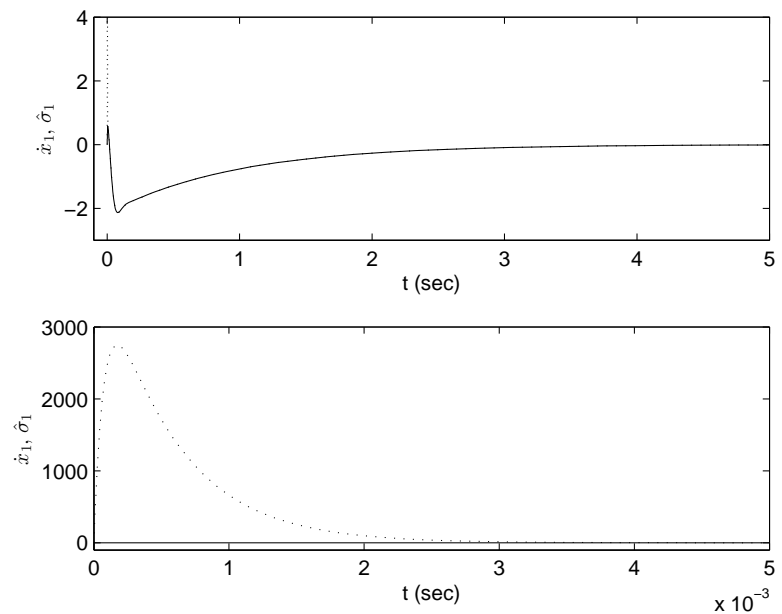

Figure 6: The solid and dotted lines represent the system state $\dot{x}_{1}$ and estimate $\hat{\sigma}_{1}$, respectively. 

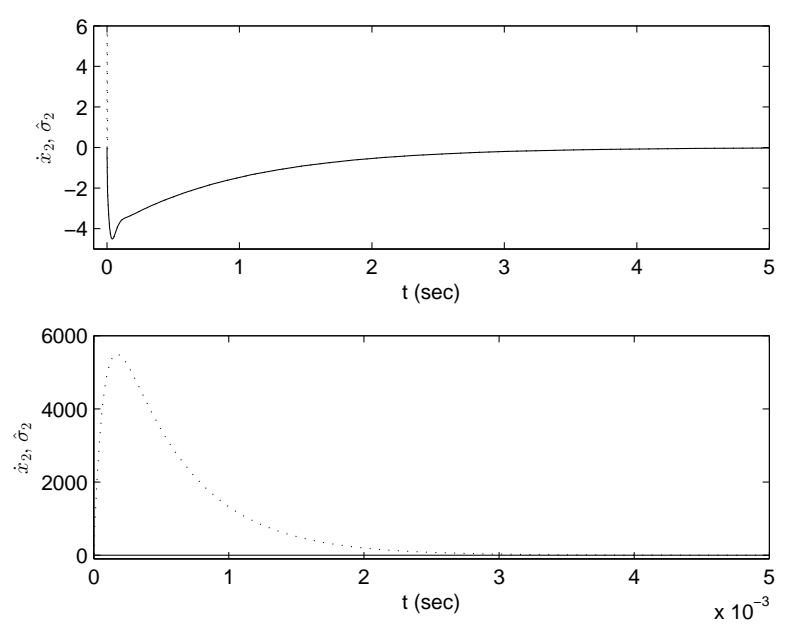

Figure 7: The solid and dotted lines represent the system state $\dot{x}_{2}$ and estimate $\hat{\sigma}_{2}$, respectively.

The results shown in Fig. 6 and 7, indicate the EHGO successfully estimates the uncertainties (i.e., the entire terms in the right-hand side of (25)) in a short period of time. At the beginning of the simulations in bottom figures of Fig. 6 and 7, the peaking phenomena are shown and quickly disappear, which are overcome by the use of saturation functions.
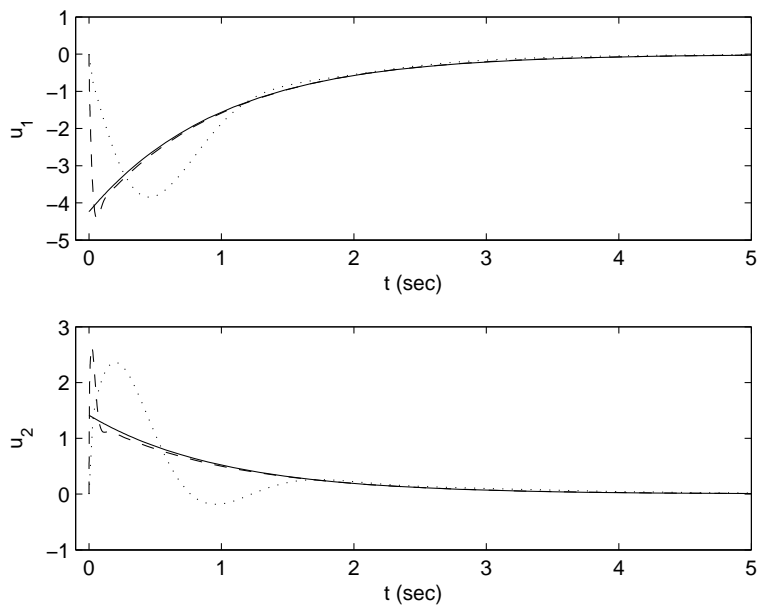

Figure 8: The solid, dashed, and dotted lines represent the inputs $u_{r}=R_{m}^{-1} x, u$ with $\mu=0.02$ and $\varepsilon / \mu=0.01$, and $u$ with $\mu=0.2$ and $\varepsilon / \mu=0.01$, respectively.

To illustrate the performances recovery for inputs, we choose the same rate of $(\varepsilon / \mu)=0.01$ with different values $\mu=0.2$ and $\mu=0.02$. The other parameters in the EHGO are the same as in (29). In Fig. 8, the input $u_{r}=R_{m}^{-1} x$ is shown with solid lines, the dashed lines represent the inputs under output feedback with parameters $\mu=0.02$ and $\varepsilon / \mu=0.01$, and the dotted lines are trajectories for the inputs under output feedback with parameters $\mu=0.2$ and $\varepsilon / \mu=0.01$. The input with the control parameters $\mu=0.02$ and $\varepsilon / \mu=0.01$, has faster convergence into $u_{r}$ than the input with control parameters $\mu=0.2$ and $\varepsilon / \mu=0.01$.

\section{Conclusions}

Unmeasured states, uncertainties, and nonaffine inputs pose challenges in control design for nonlinear systems. An output feedback control design was proposed to address these challenges. The unmeasured states and uncertainties were estimated using an EHGO and sector conditions were utilized for dynamic inversion to deal with nonaffine and uncertain inputs. The EHGO and the dynamic inversion together result in exponential convergence of the states to those of a target system. The stability and performance of the system were analyzed using singular perturbation methods and the effectiveness of the proposed controller was verified through numerical simulations. Our future work will consider extension of our approach to non-minimum phase systems.

\section{ACKNOWLEDGMENT}

This work was supported in part by the National Science Foundation under grant number ECCS-1128467.

\section{References}

Atassi, A. N., Khalil, H. K., 1999. A separation principle for the stabilization of a class of nonlinear systems. IEEE Transactions Automatic Control 44 (9), 1672 - 1687.

Back, J., Shim, H., 2008. Adding robustness to nominal output-feedback controllers for uncertain nonlinear systems: A nonlinear version of disturbance observer. Automatica 44, $2528-2537$.

Back, J., Shim, H., 2009. An inner-loop controller guaranteeing robust transient performance for uncertain mimo nonlinear systems. IEEE Transactions on Automatic Control 54, 1601 - 1607.

Chakrabortty, A., Arcak, M., 2009a. Robust stabilization and performance recovery of nonlinear systems with unmodeled dynamics. IEEE Transactions on Automatic Control 54, 1351 - 1356.

Chakrabortty, A., Arcak, M., 2009b. Time-sacle separation redesigns for stabilization and performance recovery of uncertain nonlinear systems. Automatica 45, $34-44$.

Chen, M., Ge, S. S., How, B. V. E., 2010. Robust adaptive neural network control for a class of uncertain mimo nonlinear systems with input nonlinearities. IEEE Transactions on Neural Networks 21, 796 - 812.

Freidovich, L., Khalil, H. K., November 2008. Performance recovery of feedback-linearization-based designs. IEEE Transactions on automatic control 53 (10), $2324-2334$

Ge, S. S., Zhang, J., 2003. Neural-network control of nonaffine nonlinear system with zero dynamics by state and output feedback. IEEE Transactions on Neural Networks 14, $900-918$.

Hovakimyan, N., Lavretsky, E., Cao, C., 2008a. Adaptive dynamic inversion via time-scale separation. IEEE Transactions on Neural Networks 19, 1702 $-1711$.

Hovakimyan, N., Lavretsky, E., Cao, C., December 2008b. Dynamic inversion for multivariable non-affine-in-control system via time-scale separation. International Journal of Control 81 (12), 1960 - 1967.

Hovakimyan, N., Lavretsky, E., Sasane, A. J., 2005. Dynamic inversion for nonaffine-in-control systems via time-scale separation: Part 1. In Proc. IEEE int. American Control Conference.

Khalil, H. K., 2002. Nonlinear systems. Printice Hall.

Lavant, A., 1998. Robust exact differentiation via sliding mode technique. Automatica $34,379-384$.

Lee, J., Mukherjee, R., Khalil, H., October 2012a. Control design for a helicopter using dynamic inversion and extended high gain observers. ASME Dynamic Systems and Control Conference.

Lee, J., Mukherjee, R., Khalil, H., December 2012b. Performance recovery under output feedback for input nonaffine nonlinear systems. IEEE Conference on Decision and Control. 
Tanelli, M., Punta, E., Ferrara, A., 2012. A switched second-order sliding mode control algorithm for non-affine systems with saturations. Proceedings of
Conference on Decision and Control, 5488 - 5459. 